\title{
eJRIEPS
}

Ejournal de la recherche sur l'intervention en éducation physique et sport

Numéro spécial 3 | 2020

Actes de la 11 ème Biennale de l'ARIS, Lille, 19-21 juin 2018

\section{L'utilisation de la technique des incidents critiques pour favoriser la réflexion de joueurs en sports collectifs}

Luc Nadeau, Denis Martel, Ian Poulin-Beaulieu et Joëlle Couture-Légaré

\section{CpenEdition}

Journals

Édition électronique

URL : http://journals.openedition.org/ejrieps/4336

ISSN : 2105-082

Éditeur

ELLIADD

\section{Référence électronique}

Luc Nadeau, Denis Martel, Ian Poulin-Beaulieu et Joëlle Couture-Légaré, « L'utilisation de la technique des incidents critiques pour favoriser la réflexion de joueurs en sports collectifs », eJRIEPS [En ligne], Numéro spécial 3 | 2020, mis en ligne le 01 janvier 2020, consulté le 18 février 2020. URL : http:// journals.openedition.org/ejrieps/4336

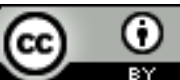

La revue eJRIEPS est mise à disposition selon les termes de la Creative Commons Attribution 4.0 International License. 


\section{L'utilisation de la technique des incidents critiques pour favoriser la réflexion de} joueurs en sports collectifs

Luc Nadeau*, Denis Martel*, lan Poulin-Beaulieu*, Joëlle Couture-Légaré ${ }^{*}$

* Département d'éducation physique, Faculté des sciences de l'éducation, Université Laval, Québec.

\section{Résumé}

L'apprentissage d'Habiletés tactiques en sports collectifs doit être basé sur la pratique de situations de jeu où les joueurs sont libres de prendre des décisions afin d'exécuter des actions appropriées au contexte. Pour ce faire, ils doivent pouvoir prendre du recul pour comprendre le jeu et choisir la meilleure option à chaque instant. Ceci est particulièrement important en sport compétitif où une mauvaise décision peut mener à la défaite. Or, le temps nécessaire pour effectuer cette réflexion est limité tant lors de matchs que lors de séances d'entraînement. Le but de cette étude est de décrire comment des joueurs ont utilisé la technique des incidents critiques (TIC) pour décrire des situations de jeu vécues lors de matchs du calendrier de compétition. Trois entraineurs ont invité leurs joueurs [football $(n=36)$ et hockey sur glace $(n=18)]$ à décrire des situations de jeu qu'ils considéraient Très efficaces (TE) ou Peu efficaces (PE)], pour le match qu'ils venaient de jouer, et de justifier leur point de vue. Les joueurs ont rapporté davantage de situations TE $(n=289)$ que PE ( $n=117)$. La majorité des récits concernaient des actions tactiques individuelles offensives liées aux principes de jeu de marquer $(n=184)$ et progresser vers le but $(n=239)$. Les constats de l'étude démontrent que l'utilisation de la TIC peut aider les joueurs à prendre $d u$ recul sur les situations vécues afin de mieux comprendre le jeu. Cette méthode aurait tout avantage à être utilisée régulièrement par les entraineurs qui privilégient une approche basée sur l'apprentissage par la compréhension du jeu.

Mots-clés: sports collectifs; compréhension du jeu; incidents critiques; principes de jeu; tactique. 


\section{Summary}

Teaching and learning tactical skills is essential to improving performance in team sport. $A$ player's understanding of the game is a valuable indication of the learning of these skills. This understanding is based on a reflection process where players need to analyse significant moment of the game. Unfortunately, the game itself, neither the exercises in practice sessions, gives enough occasions to player to be in that reflection process. The purpose of this study is to describe how competitive players can use the critical incident technique to describe tactical situations that have experienced during games. Fifty-four athletes [football $(n=36)$ and ice hockey $(n=18)$ ] were asked by their coaches to describe game situations [Very Effective (TE) and/or Less effective (NE) tactical situation] they have experienced during games. Players were free to describe and explain in detail the reasons why they considered them significant situations. A validated procedure was used to ensure an objective classification of the incidents. Players reported more $E(n=289)$ than $N E$ ( $n=117$ ) tactical situations. Majority of situations referred to individual and offensive tactical situations, in which attacking the opposite goal $(n=184)$ and progressing to the opposite goal ( $n=239)$ were most cited by players. This study suggest that the reflection process, associated with writing critical incidents, can help players and coaches to better understand the game in a Learning games through understanding approach.

Key words: team sports; game understanding; critical incidents technique; game principles; tactical skills

\section{Introduction}

De manière générale, les entraineurs en sports collectifs compétitifs proposent différentes activités d'apprentissage et de perfectionnement aux joueurs pendant les séances d'entrainement afin qu'ils améliorent leurs performances en situation de jeu (Bompa \& Carrera, 2005; Bompa \& Haff, 2009; Platonov, 1988; Garganta, 2009). Une récente étude québécoise (Nadeau, Martel, Poulin-Beaulieu et Couture-Légaré, 2017) a permis de constater que des entraineurs de hockey sur glace et de football de niveau compétitif utilisent exclusivement une approche traditionnelle d'enseignement fondée sur l'apprentissage par la répétition (Kermarrec, 2004). L'analyse de 58 séances d'entrainement a effectivement démontré la prédominance du développement d'habiletés motrices dites 


\section{eJRIEPS spécial 3 janvier 2020}

techniques (ex : pratiquer des arrêts brusques sur la glace) et/ou la réalisation de séquences de jeu précises (flow drills) où les joueurs ont peu ou aucune décision à prendre dans l'action. De surcroit, la majorité de ces activités se déroulaient sans la présence d'adversaires, impliquaient peu de joueurs à la fois et étaient axées sur l'exécution d'automatismes décontextualisés des situations réelles de jeu (Nadeau, Martel, PoulinBeaulieu \& Couture-Légaré, 2017).

\section{Problématique}

Plusieurs chercheurs remettent en question l'utilisation de l'approche d'enseignement traditionnelle pour des sports à dominante tactique comme les sports collectifs (Gréhaigne \& Godbout, 1995; Trilles, 1994). Bien que l'enseignement par la répétition ait démontré son efficacité pour l'apprentissage d'habiletés motrices simples ou encore le développement des qualités physiques, cette méthode est peu propice au développement d'actions motrices qui ne surviennent jamais de manière identique comme celles réalisées par les joueurs pendant un match (Bouthier, 1986). Les joueurs devraient donc avoir davantage d'occasions de prendre des décisions sur le jeu et mieux comprendre ce qu'il faut faire pour être efficaces. À cet égard, les joueurs se doivent d'apprendre les principes de jeu des sports collectifs qui constituent un référentiel fondé sur l'exécution appropriée d'actions tactiques pertinentes au problème de jeu vécu par un ou des joueurs au moment $X$ du jeu (Deleplace, 1979). En fait, les sports collectifs obligent les joueurs à constamment s'ajuster à la position de l'objet (ballon, rondelle, etc...) ainsi qu'aux placements et déplacements des coéquipiers et des adversaires pendant le match, tout en tenant compte de différents facteurs de jeu comme le pointage, l'enjeu de la partie, les stratégies déterminées par les joueurs et entraineurs avant le match, etc. (Gréhaigne, Godbout \& Zerai, 2014; Gréhaigne \& Nadeau, 2015). De ce fait, l'entrainement des joueurs en sports collectifs doit donc forcément favoriser l'autonomie des joueurs et leur capacité de s'adapter à chaque problème de jeu vécu (Gréhaigne \& Godbout, 1998).

Depuis quelques années, des stratégies novatrices - qui visent à permettre aux joueurs d'être davantage conscients de leurs actions et de celles des coéquipiers et adversaires afin qu'ils puissent choisir plus facilement ce qui est le plus approprié au problème de jeu rencontré (Hopper, Butler \& Storey, 2009) - sont de plus en plus employées par les entraineurs. Ces stratégies, utilisées dans plusieurs centres de formation de clubs sportifs de certains pays européens, responsabilisent davantage les joueurs à leurs apprentissages 


\section{eJRIEPS spécial 3 janvier 2020}

en utilisant des approches fondées sur la compréhension du jeu (Bunker \& Thorpe, 1982; Georget, 2013; Gréhaigne \& Godbout, 1998; Gréhaigne \& Nadeau, 2015; Nadeau, Georget, Fortier \& Godbout, 2014). Les bases de cette approche visent à ce que chaque joueur devienne autonome et responsable de son apprentissage en lui permettant de mieux comprendre les subtilités des grands principes de jeu du sport pratiqué (Frederic, Gribomont \& Cloes, 2009). À l'image des principes sur les pratiques réflexives de Dewey (repris par Chaubet, 2010), les joueurs sont amenés à réfléchir à la pertinence, la réussite et la cohérence d'actions effectuées à l'intérieur d'un cycle de réflexion (Chaubet, 2010) où ils cherchent à résoudre un problème concret du jeu pour mieux performer en se coordonnant avec leurs coéquipiers. Ces problèmes mettent en jeu des choix d'actions respectant la priorité à accorder aux grands principes du jeu et les règles d'opération qui, une fois bien compris par les joueurs, assurent une plus grande cohésion entre eux afin de gagner la partie (Gréhaigne, Godbout, \& Mahut, 1999; Nadeau et al., 2014).

Il existe différents outils et stratégies pour favoriser l'apprentissage et le perfectionnement d'athlètes en respect de cette perspective. À cet égard, la figure 1 illustre quelques outils spécifiques aux différentes composantes qui peuvent influencer l'apprentissage et les performances des athlètes (Nadeau et al., 2017). Les catégories illustrées sont communes à toute forme de paradigme de formation, autant les méthodes traditionnelles technicistes que les méthodes socio-constructivistes qui favorisent la compréhension du jeu telles que celle du Modèle de décision tactique [Tactical Decision Learning Model - TDLM] de Gréhaigne et Nadeau (2015). 


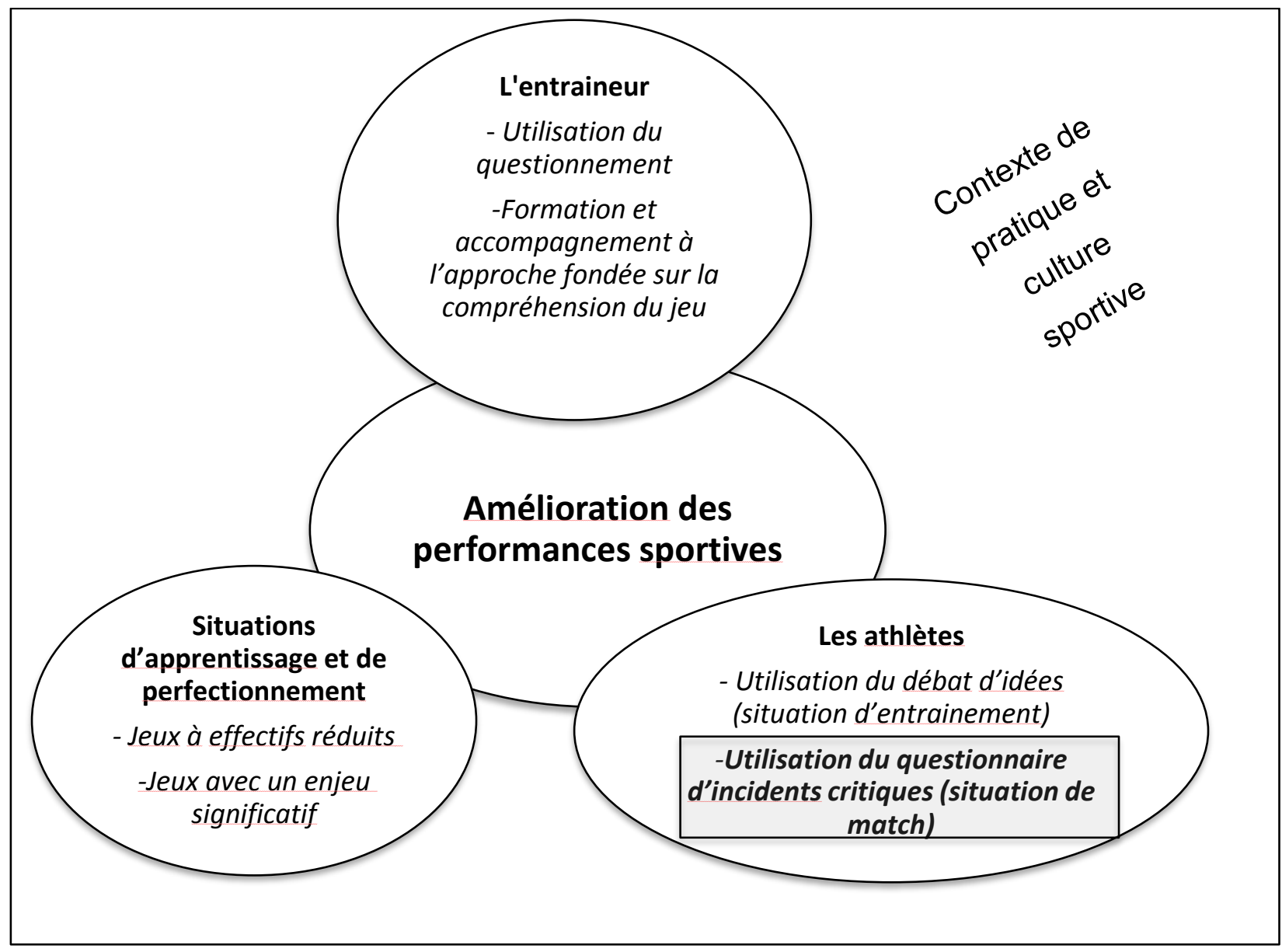

Figure 1 : Exemples d'outils utilisés pour chaque catégorie d'un modèle d'intervention d'une approche fondée sur la compréhension du jeu dans les sports collectifs (Nadeau et al., 2017)

Jusqu'à maintenant, les études qui ont traité de l'approche fondée sur la compréhension du jeu ont principalement décrit les stratégies utilisées par les joueurs et les intervenants pendant des séances d'entrainement. Par exemple, quelques études ont porté sur l'utilisation du questionnement par les entraineurs (Langevin, 1990) afin de favoriser la réflexion des joueurs pour qu'ils trouvent, par eux-mêmes, la meilleure réponse motrice au jeu effectué (Maulini, 2005). Ou encore, l'utilisation du débat d'idées - périodes de discussion entre les joueurs d'une même équipe sans l'intervention directe de l'entraineur a également fait l'objet de plusieurs études (Gréhaigne, 2007). À ce propos, l'efficacité du débat d'idées a été démontrée dans plusieurs études se déroulant dans les milieux scolaires et sportifs européens (Deriaz, Poussin \& Gréhaigne, 1998; Gréhaigne, Wallian \& BrièreGuenoun; 2015). Que ce soit pour des joueurs débutants ou même débrouillés, les discussions au sein d'équipes ( 3 à 5 joueurs) les amènent collectivement à réfléchir sur un problème de jeu particulier pour ainsi mieux comprendre le jeu et améliorer leurs 


\section{eJRIEPS spécial 3 janvier 2020}

performances (Zerai, Rezig \& Zhigbi, 2008). Par contre, cette technique s'applique plus difficilement lorsque les périodes de réflexion se limitent aux séances d'entrainement en sport compétitif. II est possible que l'apprentissage ne soit pas aussi optimal que souhaité pour tous les joueurs, particulièrement ceux qui ont toujours été habitués à attendre les indications des entraineurs ou des autres coéquipiers. En fait, pour solliciter la réflexion des joueurs sur leur performance en situation de match compétitif, le débat d'idées pendant les séances d'entrainement n'est pas toujours la meilleure stratégie à adopter. Premièrement, les occasions de débattre d'un problème de jeu directement pendant le match sont très limitées en raison d'arrêts de jeu très courts (ex. hockey sur glace) ou carrément inexistants (ex football). De plus, la période de mi-temps au football ou encore les temps d'arrêt entre les périodes au hockey sont essentiellement des moments où les entraineurs raffinent leurs consignes aux joueurs pour la suite du match ou encore où les joueurs se reposent afin d'être prêts pour la prochaine séquence de jeu (Gréhaigne, 2009). Deuxièmement, le délai entre le match réel et la séance d'entrainement peut être important et faire en sorte que les joueurs ne se souviennent plus de certains détails importants ou même de certaines séquences de jeu. Troisièmement, les discussions pendant les débats d'idées sont souvent animées par certains joueurs et concernent surtout des problèmes de jeu collectifs. Ces débats n'amènent donc pas toujours les joueurs à réfléchir individuellement sur leur propre compréhension du jeu en fonction de leurs habiletés, de leurs rôles au sein de l'équipe, etc. Finalement, l'utilisation du débat d'idées pendant un match régulier au niveau compétitif s'avère difficile à mettre en œuvre compte tenu du jeu en continu, des équipements, de l'intensité ou même de la nature du jeu.

Malgré tout, c'est à partir de différentes activités de réflexion que les joueurs apprennent le jeu et déterminent quelles sont les meilleures actions à prendre afin de vaincre les adversaires (Gréhaigne et al., 2015). À ce propos, Chaubet (2010, p. 64) mentionne que : " la réflexion est un processus d'investigation déclenché par un élément interpellant, qui aboutit à sa reconceptualisation et peut avoir des effets psychologiques sur l'acteur ou pragmatiques sur l'action ». À notre connaissance, aucune étude n'a été menée jusqu'à maintenant pour analyser les stratégies que peuvent utiliser des joueurs - en dehors des séances d'entrainement - qui peuvent susciter leur réflexion sur des situations de jeu. Or, des retours individuels sur un match, au-delà d'un processus de réflexion en équipe, peuvent s'avérer très bénéfiques pour la compréhension du jeu en raison du caractère marquant des situations retenues par le joueur et de liens avec des conséquences directes 


\section{eJRIEPS spécial 3 janvier 2020}

(positives comme un but marqué ou négatives comme la perte de possession du ballon à l'adversaire). La réflexion et la prise de recul par rapport à la manière de jouer en sports collectifs demeurent toutefois les éléments centraux d'une approche fondée sur la compréhension du jeu (Azzarito, Solomon \& Afeman, 2003). C'est grâce à ce moment de recul que le joueur peut analyser le résultat des actions effectuées antérieurement et tenter de comprendre quelles seraient les meilleures façons de vaincre l'adversaire et de collaborer avec ses coéquipiers à l'avenir. Or, cette réflexion n'est pas nécessairement automatique et assurée à chaque occasion de jeu. C'est particulièrement vrai lorsque le joueur est devant un problème complètement nouveau ou une situation pour laquelle il n'a aucune idée des moyens pour s'en sortir. Pour ce faire, la période de réflexion doit d'abord s'organiser autour des éléments marquants pour ce joueur, des éléments où il reconnait certains indices de jeu qui l'aideront à développer progressivement sa compréhension comme par exemple les actions qui ont fait progresser le jeu ou qui ont mené à un tir au but. Certaines situations, qu'elles soient positives ou négatives, resteront ancrées dans la mémoire du joueur si celui-ci les considère plus décisives (Gerbert \& Durny, 2013). Ces situations sont donc des éléments clefs qui peuvent être déclencheurs d'un processus de régulation de ses actions et décisions en situation de match. II s'avère donc important de trouver d'autres méthodes qui vont permettre aux joueurs de prendre conscience de ce qu'ils ont fait dans le match et des problèmes rencontrés. Ces réflexions devraient mener vers des recherches de solutions qu'ils pourront tenter d'expérimenter lors des séances d'entrainements d'une part et, encore plus important, dans les prochains matchs. La prémisse importante est nécessairement leur désir de s'améliorer et d'améliorer leurs performances (Zerai et al., 2008).

Le présent article est tiré d'une recherche-action destinée à influencer le modèle de formation préconisé par des entraineurs de sports collectifs (Nadeau, Martel, PoulinBeaulieu \& Légaré-Couture, non publié). Plus précisément, cette étude invitait des entraineurs de football et de hockey sur glace à utiliser la technique des incidents critiques afin de placer davantage les athlètes au centre de leur processus d'apprentissage.

\subsection{Le questionnaire des incidents critiques}

La technique des incidents critiques est une méthode permettant le recueil des perceptions et elle est utilisée principalement dans le domaine de la psychologie (Flanagan, 1954). Selon Dant (1995), cette technique suscite la réflexion chez les participants en plus de 


\section{eJRIEPS spécial 3 janvier 2020}

fournir des données intéressantes à l'intervenant sur leurs perceptions de situations ou d'évènements marquants (Amade-Escot, 2005).

L'utilisation d'un questionnaire des incidents critiques après les matchs devient alors un outil intéressant pour permettre au joueur de structurer ses réflexions et de l'amener à prendre du recul par rapport à ses actions. En effet, l'incident critique révèle un évènement précis et marquant, habituellement imprévu, comportant un enjeu important aux yeux du participant et mettant en évidence une compétence ou problématique particulière (Lescarbeau et al., 1996). Cette prise de conscience d'un évènement est la première étape du cycle de réflexion de Dewey (1933), qui tel que repris par Chaubet (2010, p. 63), invite un joueur :

1) à l'identification d'une « situation interpelante » qui sert d'amorce à sa réflexion ;

2) à une investigation par l'observation ;

3) à une analyse ;

4) à une restructuration de sa compréhension initiale (ou « reconceptualisation») ;

5) à l'adoption d'une action différente lors d'une situation de jeu ultérieure.

De toute évidence, plusieurs actions tactiques pendant les matchs possèdent ces caractéristiques. Ces moments déterminants pour le joueur, et la prise de conscience du contexte de la situation (où je suis, où est le ballon, où sont mes coéquipiers et les adversaires, quelles étaient mes sensations, quelles sont les priorités de jeu, les probabilités de réussite, les conséquences en cas d'échec, etc.), peuvent potentiellement l'aider à développer sa compréhension du jeu et des choix qu'il peut faire pour mieux coordonner ses actions à celles de ses coéquipiers contre les actions des joueurs d'une équipe adverse.

L'utilisation de la technique des incidents critiques a le potentiel d'apporter deux grands avantages aux joueurs et entraineurs pour la compréhension du jeu : 1) le processus de réflexion devient systématique pour le joueur et 2) l'entraineur peut avoir accès aux réponses des joueurs afin de mieux comprendre leur raisonnement. Pour l'entraineur, cette méthode peut l'aider à accompagner les joueurs à trouver des solutions possibles ou amorcer des discussions de clarification sur les décisions et actions à prendre sur le jeu. De plus, la lecture des incidents rapportés peut mener vers la création de situations d'apprentissage plus spécifiques lors des entrainements. Ainsi, l'entraineur connait davantage le niveau de compréhension de ses joueurs et peut lui-même en apprendre plus sur le jeu à partir de la lecture des questionnaires (Cloes \& Pieron, 1989). 


\section{Méthodologie}

\subsection{Les participants}

Deux entraineurs masculins en Football (U-16 Féminin - division 1; Sénior masculin division 2) et un entraineur masculin en Hockey sur glace (Niveau juvénile - division 2) ont participé au projet lors d'une saison entière de compétition. La répartition des joueurs (14 ans $\leq \mathrm{x} \leq 21$ ans) selon les équipes est présentée au Tableau I.

Tableau I : Répartition des joueurs selon les équipes

\begin{tabular}{lc} 
Équipe & Nombre de joueurs \\
\hline Hockey Juvénile M div 2 & 18 \\
Football U-16 F division 1 & 21 \\
Football Sénior M division 2 & 15 \\
\hline Total & 54 \\
\hline
\end{tabular}

\subsection{Le Questionnaire des incidents critiques (QIC)}

Le Questionnaire des incidents critiques (QIC) invitait les joueurs à décrire de manière détaillée des situations tactiques jugées Très efficaces ou Peu efficaces puis à justifier leur point de vue. Les directives inscrites sur le questionnaire, respectivement pour une situation tactique jugée Très efficace ou Peu efficace par un joueur, sont présentées ci-après dans l'encadré. Ces informations visaient à favoriser un processus de réflexion des joueurs davantage basé sur des faits, et à fournir aux entraineurs des informations clefs pour se remémorer - dans la mesure du possible - l'incident rapporté par le joueur. Dans certains cas, les entraineurs ont été en mesure de les repérer sur la vidéo de matchs filmés et de les utiliser lors d'une discussion avec le joueur. Le QIC offrait également la possibilité aux joueurs de dessiner de manière schématique le lieu sur la surface de jeu (Zone défensive Zone neutre - Zone offensive), les joueurs concernés (Partenaires et/ou adversaires), le rôle des joueurs (Porteur ou Non-porteur) ainsi que leurs déplacements et actions au fil de l'incident. Ces schémas ont été utilisés par les chercheurs afin d'assurer une juste compréhension du contenu des évènements rapportés par les joueurs. Par ailleurs, précisons qu'un joueur pouvait raconter autant de situations tactiques - Très efficaces ou Peu efficaces - que désiré à la suite d'un match. 


\section{Directives pour la description de situations tactiques}

\section{Situation tactique jugée Très efficace}

Au cours du dernier match, indique une situation où tu juges avoir pris une bonne décision tactique pendant le jeu. Précise la situation, les joueurs qui étaient dans ton champ de vision, le pointage, le temps du jeu et tous les détails qui y sont reliés et dont tu te souviens.

Indique également pourquoi tu considères qu'il s'agissait d'une bonne décision tactique.

\section{Situation tactique jugée Peu efficace}

Au cours du dernier match, indique une situation où tu juges avoir pris une mauvaise décision tactique pendant le jeu. Précise la situation, les joueurs qui étaient dans ton champ de vision, le pointage, le temps du jeu et tous les détails qui y sont reliés et dont tu te souviens.

Indique également pourquoi tu considères qu'il s'agissait d'une mauvaise décision tactique.

II est à noter que les joueurs pouvaient également mentionner des aspects techniques du jeu comme évènement marquants mais ces aspects devaient être reliés à une situation particulière de jeu impliquant coéquipiers et adversaires.

\subsection{La procédure d'utilisation du QIC par les joueurs}

Deux procédures distinctes ont été mises en place par les entraineurs pour inviter leurs joueurs à utiliser le QIC. Au football, le QIC était proposé sous la forme d'un cahier personnalisé que les joueurs conservaient dans leur sac de sport. Cette procédure leur offrait la possibilité de rédiger des incidents critiques après chacun des matchs de la saison, à un moment jugé opportun. Les entraineurs consultaient les cahiers à raison de deux à trois fois par mois afin de prendre connaissance du contenu des incidents rapportés et de l'analyse réalisée par les joueurs. Au hockey sur glace, la procédure instaurée permettait aux joueurs de rédiger des incidents critiques uniquement pour des matchs sélectionnés par l'entraineur et ce, immédiatement après ledit match lors des déplacements en autobus. 


\section{eJRIEPS spécial 3 janvier 2020}

Ainsi, les QIC étaient récupérés dans les jours après le match et conservés par l'entraineur. Les membres de l'équipe de recherche ont recueilli une copie des QIC complétés par les l'ensemble des joueurs (Football et Hockey sur glace) à différents moments de la saison de compétition.

\subsection{L'analyse des situations tactiques rapportées}

Les situations tactiques rapportées $(\mathrm{N}=406)$ par les joueurs ont fait l'objet d'une analyse de contenu à modèle fermé (L'Écuyer, 1987) inspirée des principes de jeu de base des sports collectifs interpénétrés (Figure 2) de Werner, Thorpe et Bunker (1996).

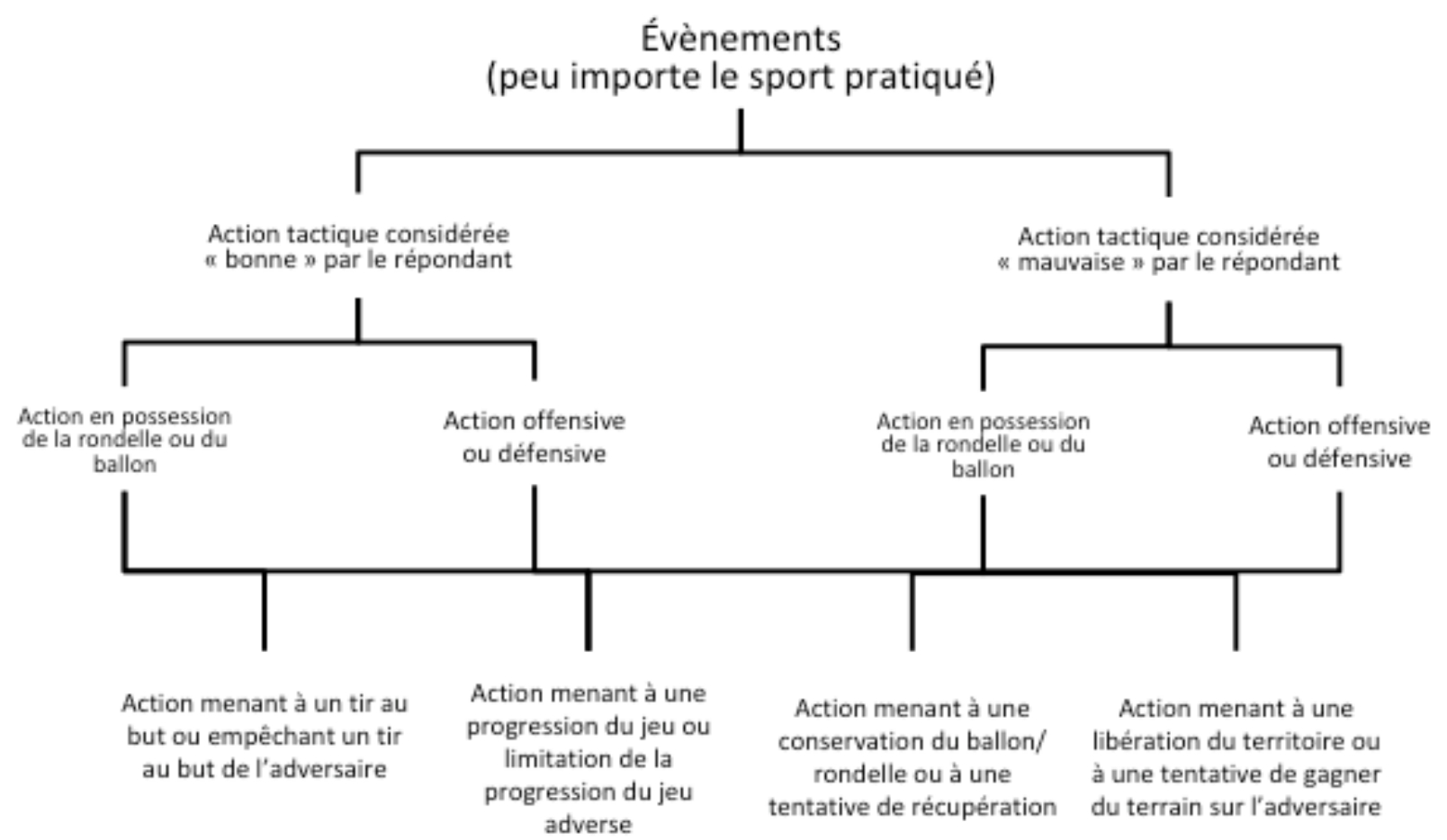

Figure 2 : Principes de jeu utilisés pour l'analyse du contenu des situations tactiques

Pour ce faire, le chercheur identifiait l'ensemble des principes et règles tactiques contenus de manière explicite dans le récit du joueur. Par exemple, dans la situation Peu efficace rapportée ci-après, quatre principes de jeu [Caractère gras] peuvent être identifiés :

« Je viens de récupérer le ballon [Reprise de possession]. Un de mes coéquipiers (défenseur gauche) fait un appel en profondeur. Je décide donc de le rejoindre par la voie des airs [Progression du ballon]. Toutefois, ma passe n'est pas assez précise, je me fais intercepter 


\section{eJRIEPS spécial 3 janvier 2020}

[Perte de ballon/conservation]. J'aurais dû jouer avec mes défenseurs pour conserver le ballon [Conservation du ballon] et non le perdre bêtement ».

Par choix, les mentions d'éléments plus techniques ou liés à la condition physique n'ont pas été spécifiquement répertoriés afin de mettre davantage d'emphase sur les aspects tactiques du jeu. Par exemple, la mention d'une exécution technique fautive lors d'une passe menant à une interception était classée sous le principe tactique Perte de ballon/conservation. L'analyse de contenu des situations tactiques et l'établissement de sa validité ont été réalisées en trois étapes. Lors de la première étape, un chercheur et deux assistants ont d'abord classifié la totalité des situations recueillies sur la base d'un consensus inter-analystes. À la deuxième étape, une base de données a été créée (MS Excel) en indiquant pour chacune des situations analysées les informations suivantes : le numéro du joueur, le sport, l'équipe, l'âge du joueur, le type de situation (Très efficace ou Peu efficace) et, s'il y a lieu, les actions des coéquipiers et ou des adversaires et, enfin, les principes de base (Figure 2). À la troisième étape, un expert en sport collectif - non impliqué dans la catégorisation initiale des situations - a été invité à analyser 40 situations pré-codées lors de la première étape. Ce dernier, préalablement formé aux règles de codification, à la procédure de codage et à la saisie des informations, a obtenu un pourcentage d'accord de $92 \%$.

\section{Résultats}

Au total, les joueurs ont rapporté davantage d'actions tactiques dites Très efficaces ( $\mathrm{N}=289)$ que d'actions tactiques dites Peu efficaces ( $N=117$ ) (Tableau II). Les joueurs masculins de football et de hockey sur glace sont ceux qui rapportent un pourcentage plus élevé d'actions tactiques jugées Peu efficaces (Football, 40,5\%; Hockey sur glace, 39,3\%). À l'opposé, les joueuses de football (Catégorie U-16) rapportent un pourcentage très élevé d'actions tactiques jugées Très efficaces (79,1\%). 
Tableau II : Nombre d'actions tactiques jugées Très efficaces ou Peu efficaces

\begin{tabular}{lccc}
\hline \multicolumn{1}{c}{ Équipe } & $\begin{array}{c}\text { Nombre } \\
\text { de } \\
\text { joueurs }\end{array}$ & $\begin{array}{c}\text { Nombre d'actions } \\
\text { tactiques Très efficaces }\end{array}$ & $\begin{array}{c}\text { Nombre d'actions } \\
\text { tactiques Peu efficaces }\end{array}$ \\
\hline Hockey Juvénile M div 2 & 18 & $34(60,7 \%)$ & $22(39,3 \%)$ \\
Football U-16 F division 1 & 20 & $189(79,1 \%)$ & $50(20,9 \%)$ \\
Football Senior M division 2 & 15 & $66(59,5 \%)$ & $45(40,5 \%)$ \\
\hline Total & 53 & $289(71,1 \%)$ & $117(28,8 \%)$ \\
\hline
\end{tabular}

De manière générale (Tableau III), les principes offensifs (481 mentions) sont nettement plus évoqués par les joueurs que les principes défensifs (156 mentions). Plus spécifiquement, les principes de base offensifs Marquer (184 occasions) et Faire progresser le jeu (239 occasions) ont été majoritairement évoqués par les joueurs dans leurs récits. Pour les actions tactiques défensives, les principes Reprendre possession (64 occasions) et Limiter la progression (44 occasions) comptent parmi les plus fréquemment invoqués par les joueurs.

Tableau III : Fréquence des principes de jeu identifiés au sein des actions tactiques rapportées

\begin{tabular}{|c|c|c|c|c|c|c|c|c|}
\hline \multirow[b]{2}{*}{ Équipes } & \multicolumn{4}{|c|}{ Principes offensifs } & \multicolumn{4}{|c|}{ Principes défensifs } \\
\hline & Marquer & $\begin{array}{c}\text { Faire } \\
\text { progresser }\end{array}$ & Conserver & $\begin{array}{l}\text { Gagner } \\
\text { du } \\
\text { territoire }\end{array}$ & $\begin{array}{c}\text { Empêcher de } \\
\text { marquer }\end{array}$ & $\begin{array}{l}\text { Limiter la } \\
\text { progression }\end{array}$ & $\begin{array}{l}\text { Reprendre } \\
\text { possession }\end{array}$ & $\begin{array}{l}\text { Libérer le } \\
\text { territoire } \\
\text { défensif }\end{array}$ \\
\hline Hockey & & & & & & & & \\
\hline $\begin{array}{r}\text { Juvénile M } \\
\text { division } 2\end{array}$ & 20 & 16 & 10 & 2 & 12 & 10 & 9 & 3 \\
\hline $\begin{array}{r}\text { Football U-16 } \\
\text { F division } 1\end{array}$ & 133 & 166 & 32 & 4 & 7 & 13 & 41 & 5 \\
\hline $\begin{array}{r}\text { Football } \\
\text { senior } \mathrm{M} \\
\text { division } 2\end{array}$ & 31 & 57 & 9 & 1 & 20 & 21 & 14 & 1 \\
\hline Total & 184 & 239 & 51 & 7 & 39 & 44 & 64 & 9 \\
\hline
\end{tabular}

Par ailleurs, la grande majorité des joueurs ont rapporté leurs actions individuelles ou celles de leurs coéquipiers plutôt que celles effectuées par les adversaires (Tableau IV) dans leurs descriptions. En fait, $83,5 \%$ des actions tactiques rapportées concernent des situations où les actions des joueurs adverses ne sont nullement mentionnées. Notons que les joueurs ont indiqué à la fois leurs actions et/ou celles de leurs coéquipiers ainsi que les actions de 


\section{eJRIEPS spécial 3 janvier 2020}

leurs adversaires dans seulement $5,4 \%$ des cas. Ce constat laisse supposer que les joueurs de l'étude ne transcrivaient pas les actions de leurs adversaires, étaient moins préoccupés par ces actions ou ne les prenaient pas en compte lors de leur processus de réflexion.

Tableau IV : Fréquence des actions considérées lors de la description des actions tactiques

\begin{tabular}{lccc}
\hline & $\begin{array}{c}\text { Actions } \\
\text { individuelles et } \\
\text { Équipe }\end{array}$ & $\begin{array}{c}\text { Actions des } \\
\text { adversaires }\end{array}$ & $\begin{array}{c}\text { Actions individuelles, des } \\
\text { coéquipiers et des } \\
\text { adversaires, }\end{array}$ \\
\hline Hockey Juvénile M div 2 & $44(78,6 \%)$ & $12(21,4 \%)$ & $0(0 \%)$ \\
Football U-16 F division 1 & $207(86,6 \%)$ & $10(4,2 \%)$ & $22(9,2 \%)$ \\
Football Senior M division 2 & $88(79,3 \%)$ & $23(20,7 \%)$ & $0(0 \%)$ \\
\hline Total & $339(83,5 \%)$ & $45(11,1 \%)$ & $22(5,4 \%)$ \\
\hline
\end{tabular}

Les QIC complétés par les joueurs révèlent différents niveaux de description des situations rapportées. Dans l'exemple ci-après, un niveau de rédaction dit descriptif- lequel a été le plus fréquemment observé - fait état d'un texte et d'un schéma relativement simples qui concernent essentiellement le mouvement d'un joueur et du ballon. Chez le débutant, l'intérêt et l'attention porte essentiellement sur le ballon. Plus le joueur apprend et évolue, plus il devient conscient de la présence des coéquipiers et adversaires et donc de la configuration de jeu. Or, les joueurs illustraient rarement la position de plus de 3 à 4 joueurs sur le schéma, ce qui semble plutôt normal selon le calibre de jeu, et qui correspond à un niveau de conscience de premier niveau des joueurs sur le terrain en sports collectifs (Caty et al., 2007). 


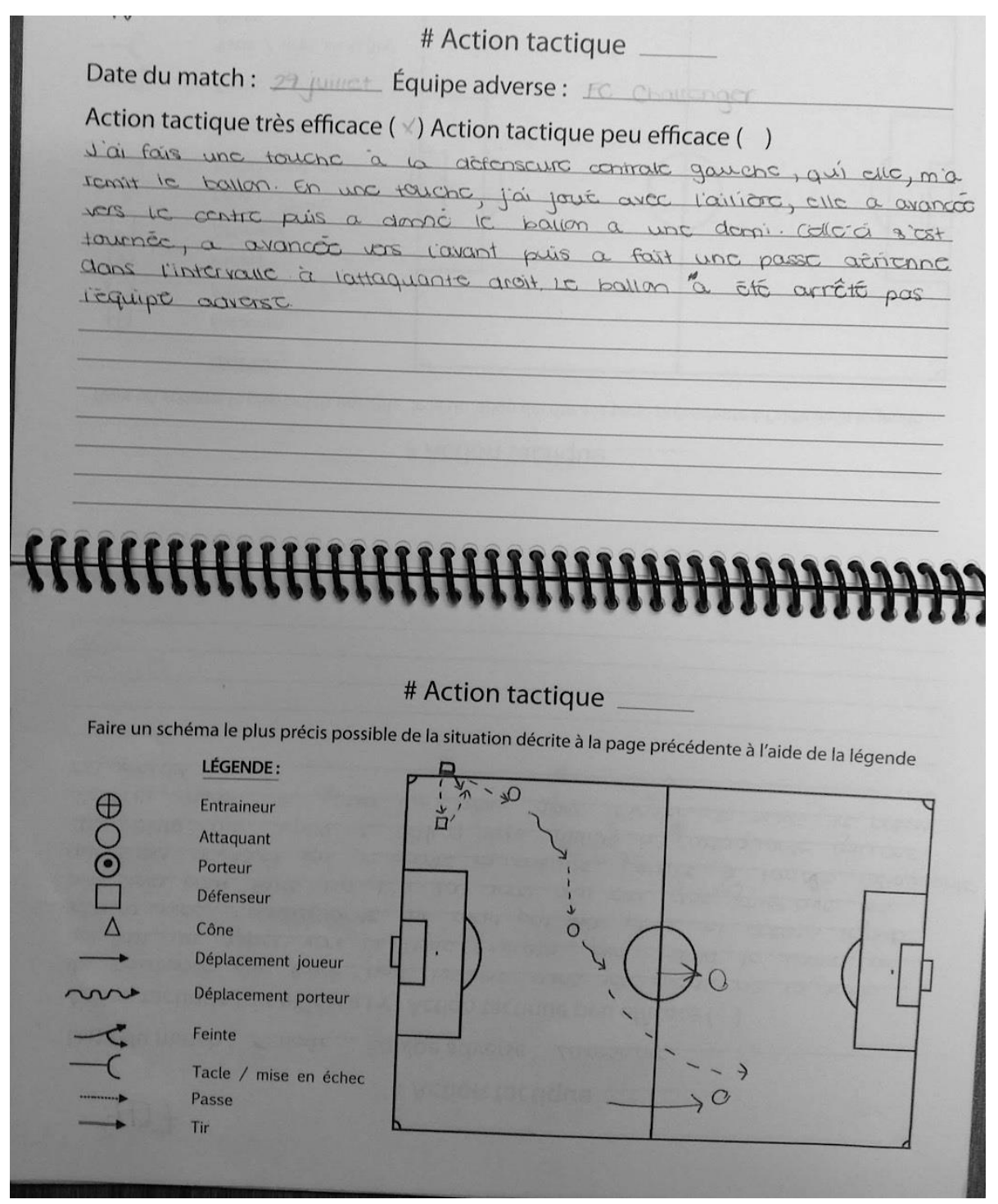

Figure 3 : QIC illustrant un niveau de réflexion dit descriptif

En certaines occasions, des joueurs ont décrit leur incident de manière plus détaillée tout en précisant les raisons qui justifiaient l'efficacité ou non de leur action sur le terrain. L'exemple ci-après (Figure 4) laisse supposer que le joueur a réfléchi à la situation de jeu et à ses actions qu'il justifie par le respect d'un principe de jeu d'équipe offensif (jouer vers l'avant) tout en étant conscient des conséquences possibles sur l'aspect défensif ( « j'avais le temps et l'espace pour le faire »). 


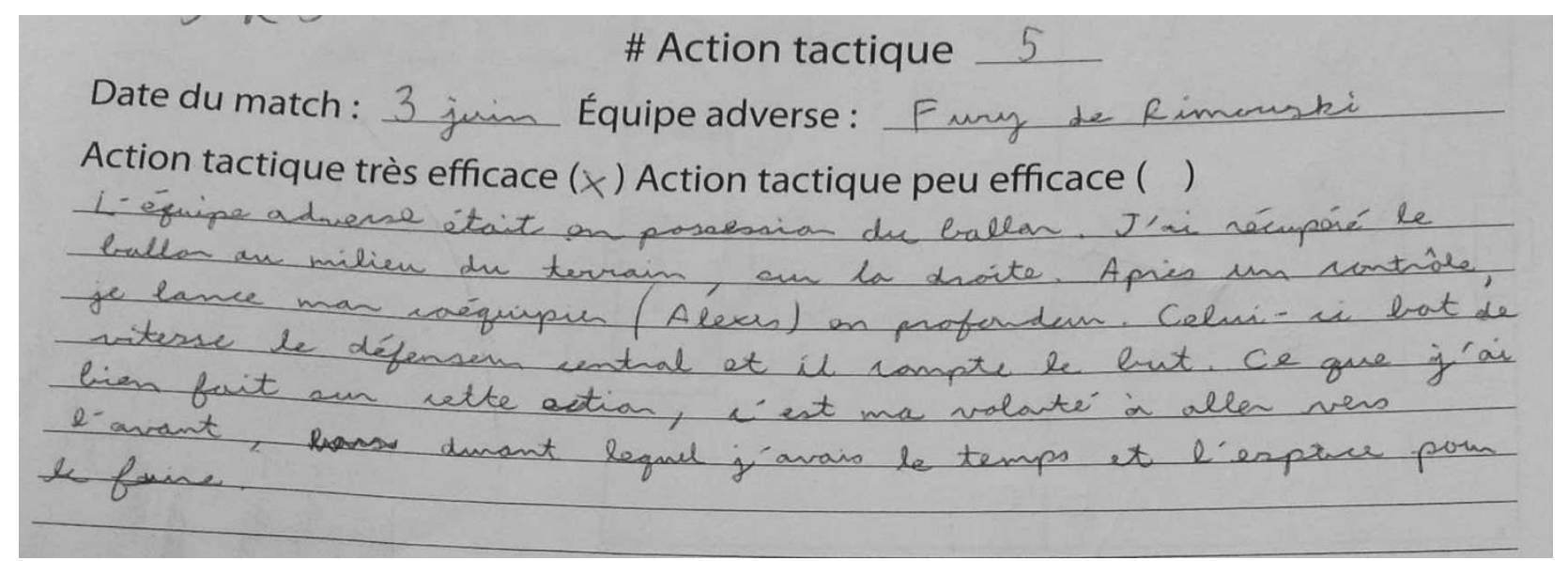

Figure 4 : Exemple de réponse décrivant une situation de jeu efficace et la justification

Enfin, dans de rares occasions, des joueurs ont décrit des situations où ils ont anticipé les actions de leurs coéquipiers ou adversaires (surlignées en gris) :

"L'action tactique très efficace que j'ai fait pendant le match est survenue vers la fin de la deuxième demie. L'action a débuté d'une passe à Ariane par une joueuse de notre équipe (mais je ne me souviens plus de qui). Par la suite, Ariane est partie vers le but, en contrôle du ballon et s'est rendue environ jusqu'à la ligne de but. Par la suite, elle a tiré au but mais comme elle n'avait plus d'angle, la gardienne l'a arrêté mais a donné un retour. Lorsque j'ai vu Ariane accélérer avec le ballon, j'ai sprinté au but. Lorsqu'il y a eu le retour, j'étais juste devant la gardienne. J'ai donc amorti le ballon qui était dans les airs et j'ai frappé du côté opposé à la gardienne. Par contre, une défenseure de l'autre équipe a réussi à frapper le ballon encore dans les airs se dirigeant vers le but mais au lieu de le dégager, elle a rentré le ballon dans la lucarne de son but ».

Nous pensons que ce dernier exemple met en lumière un meilleur niveau de compréhension du jeu qui va au-delà d'une simple description de la situation de jeu vécue. L'anticipation du jeu des coéquipiers et des adversaires constitue un avantage certain car elle permet de prendre une avance sur ce qui peut arriver et donne davantage de temps pour analyser les options possibles afin de prendre une décision plus pertinente. Cette notion d'anticipation était également parfois présente lorsque les joueurs utilisaient l'expression « j'ai vu que... » comme dans l'exemple suivant : 


\section{eJRIEPS spécial 3 janvier 2020}

"En fin de deuxième période, j'étais sur la bande (balustrade) dans notre zone en sortie de zone, j'ai reçu la rondelle et j'ai vu que le défenseur adverse allait me pincher (mettre en échec) et que StGermain coupait au centre, alors je l'ai chippé (envoyé dans les airs) sur la bande et ils (Émile et Paul-Olivier) ont eu un 2 contre 1 ».

\section{Discussion}

Les évènements recueillis révèlent presque tous que les joueurs eux-mêmes sont habituellement le point de départ de l'action décrite ou, à quelques occasions, le point terminal (ceux qui ont marqué le but par exemple). Les évènements qu'ils retiennent davantage sont ceux où ils sont personnellement impliqués dans l'action et en possession de l'objet (rondelle, ballon), ce qui représenterait, selon Bell, Kelton, McDonagh, Mladenovic et Morrison (2011), un premier niveau de réflexion et de compréhension du jeu (je pense à mes propres actions). Ainsi, les joueurs de football et de hockey sur glace qui ont participé à cette étude ont davantage décrit ce qu'ils ont fait durant certaines phases de jeu et semblent encore peu conscients (ou préoccupés) des actions tactiques que l'on pourrait qualifier de deuxième niveau (ex : ce que Julien fera si je lui fais la passe) et même d'un troisième niveau (ex : si le ballon se rend à Marc ET que l'adversaire lui ferme l'intervalle, je pourrais me déplacer vers la gauche dans l'espace libre qui sera créé et ainsi être démarqué). Pourtant, ces réflexions considérées de deuxième ou de troisième niveau tactique sont davantage liées à l'anticipation du jeu et ainsi à la reconnaissance des mouvements et déplacements des joueurs et les configurations de jeu, dont certaines sont prototypiques (Caty, Meunier, \& Gréhaigne, 2007). Selon Gréhaigne (2007), la reconnaissance de ces configurations prototypiques est le point de départ d'une compréhension de jeu.

Par ailleurs, une certaine logique des principes de jeu a été respectée en démontrant que les évènements qui ont mené à marquer des points et à faire progresser le jeu pour marquer des points sont déterminants pour les joueurs des sports collectifs. Ces aspects sont plus valorisés et sont donc plus marquants que les actions défensives, surtout pour les jeunes joueurs. On remarque toutefois que les joueurs plus âgés (séniors) mentionnent davantage d'évènements " défensifs ", ce qui pourrait s'expliquer par une meilleure compréhension de leur rôle sur le jeu au sein d'une équipe. 


\section{eJRIEPS spécial 3 janvier 2020}

Comme mentionné, l'importance qu'accorde un entraineur à la réflexion des joueurs est déterminante pour favoriser leur participation à ce processus. Dans deux cas sur trois, les entraineurs ont en quelque sorte abandonné le soutien aux joueurs, prétextant un manque de temps et un niveau d'intérêt mitigé de leurs joueurs, surtout chez les garçons. À ce propos, il est possible que la culture sportive explique en bonne partie la réaction des joueurs et entraineurs récalcitrants. En fait, il est plausible de croire que la réaction des joueurs aurait été différente si ce genre d'exercice avait été instauré à un plus jeune âge et avec un meilleur accompagnement des entraineurs. Malgré tout, les trois entraineurs ont confirmé l'utilité de la TIC et de sa contribution pour apprécier la compréhension du jeu de leurs joueurs. Ils ont également souligné que c'est à partir des incidents rapportés qu'ils ont pu amorcer une discussion avec leurs joueurs afin d'échanger sur leur vision respective du jeu. Néanmoins, la plupart des entraineurs avaient beaucoup tendance à «être la référence " et donner leurs propres solutions de jeu au lieu de pousser plus loin la réflexion du joueur face à un problème de jeu en particulier.

Finalement, nous estimons que les joueurs doivent être accompagnés de manière systématique et soutenue lors d'une telle démarche de réflexion. Un entraineur a tout intérêt à lire et discuter des évènements décrits par les joueurs en suscitant davantage de précision et de justifications de leur part. Questionner davantage les joueurs, leur demander les raisons du choix des évènements, demander « quelle aurait été une meilleure solution selon toi? ", aurait contribué à mieux faire réfléchir les athlètes et à les amener vers la recherche de solutions.

\section{Conclusion}

Les approches fondées sur la compréhension du jeu dans les sports collectifs doivent s'appuyer sur un processus de réflexion systématique à partir des situations de jeu réelles vécues par les joueurs. Bien que des méthodes comme les débats d'idées soient avantageuses pour amener les joueurs à mieux comprendre le jeu lors de situations d'entrainement, cette stratégie peut difficilement être utilisée pendant des matchs de football ou de hockey sur glace en situation réelle. L'utilisation d'un questionnaire d'incidents critiques en relation avec le dernier match joué pourrait être une stratégie pertinente et réaliste pour susciter la réflexion des joueurs.

L'étude effectuée révèle que les joueurs retiennent davantage des situations où ils sont au cœur de l'action en possession de l'objet (ils amorcent ou terminent la séquence) et que leurs préoccupations portent davantage sur les actions efficaces offensives menant à une 


\section{eJRIEPS spécial 3 janvier 2020}

occasion de marquer ou de faire progresser le jeu pour marquer. Toutefois, l'efficacité de l'utilisation de cette méthode repose sur un support concret de l'entraineur auprès des joueurs et sur sa capacité à exiger d'eux une réflexion autonome qui mène à l'identification de solutions à des problèmes de jeu significatifs. De plus, la qualité de la structure de l'organisation de l'équipe de même que l'importance qu'accorde l'entraineur aux moments de réflexion sont cruciales au succès de l'utilisation de cette méthode. II nous apparait donc important d'intégrer l'utilisation du questionnaire aux activités régulières de l'équipe. L'entraineur doit accorder le temps nécessaire pour interagir avec les joueurs afin de comprendre leurs préoccupations, souligner l'importance de compléter le questionnaire régulièrement et éviter de juger de la nature de leurs réflexions.

\section{Références}

Amade-Escot, C. (2005). Using the critical didactic incidents method to analyze the content taught. Journal of Teaching in Physical Education, 24, 127-148.

Azzarito, L., Solomon, M. A., \& Afeman, H. (2003). Investigating a constructivist approach in physical education: Bridging theory and practice. Research Quarterly for Exercise and Sport, 74(1), 35-36.

Bell, A., Kelton, J., McDonagh, N., Mladenovic, R., \& Morrison, K. (2011). A critical evaluation of the usefulness of a coding scheme to categorize levels of reflective thinking. Assessment \& Evaluation in Higher Education, 36(7), 797-815.

Bompa, T., \& Carrera, M. (2005). Periodization training for sports. Champaign, IL: Human Kinetics.

Bompa, T., \& Haff, G. (2009). Periodization: Theory and Methodology of Training. Champaign, IL : Human Kinetics.

Bouthier, D. (1986). Comparaison expérimentale des effets de différents modèles didactiques des sports collectifs. In E.P.S. contenus et didactique (pp. 85-89). Paris: SNEP.

Bunker, D. \& Thorpe, R. (1982). A model for the teaching of games in the secondary schools. Bulletin of Physical Education, 10, 9-16.

Caty, D., Meunier, J.-N., \& Gréhaigne, J.-F. (2007). Modélisations des attaques réussies pour progresser dans les sports collectifs en EPS. Spirale-Revue de recherches en éducation. 40. 105-115. doi.org/10.3406/spira.2007.1397

Chaubet, P. (2010). Saisir la réflexion pour mieux former à une pratique réflexive : d'un modèle théorique à son opérationnalisation. Éducation et francophonie, 38 (2). https://doi.org/10.7202/1002164ar.

Cloes, M., \& Piéron, M. (1989). Identification de comportements enthousiastes de l'enseignant perçus par des élèves lors de séances d'éducation physique. Revue de l'Education Physique, 29 (1), 7-16.

Dant, W. (1995). Using critical incidents as a tool for reflection. In S. M. Fowler, and M. G. Mumforf (Eds.), Intercultural Sourcebook: Cross-cultural training methods (pp. 141146). Yarmouth, MN: Intercultural Press. 


\section{eJRIEPS spécial 3 janvier 2020}

Deleplace, R. (1979). Rugby de mouvement - Rugby total. Paris : Éducation Physique et Sport.

Deriaz, D., Poussin, B., \& Gréhaigne, J.-F. (1998). Le débat d’idées. Revue EPS, 273, 8082.

Flanagan, J. C. (1954). The critical incident technique. Psychological Bulletin, 51, 327-358.

Frédéric, O., Gribomont, J., \& Cloes, M. (2009). Comparaison de strategies d'entrainement du basketball en milieu scolaire et en milieu sportif. eJRIEPS, 16, 6-21.

Garganta, J. (2009). Trends of tactical performance analysis in team sports: bridging the gap between research, training and competition. Revista Portuguesa de Ciências do Desporto, 9(1), 81-89.

Georget, S. (2013). La mesure de la performance avec et sans ballon des joueurs d'un programme intensif scolaire de football (Mémoire de maitrise). Retrieved from http://hdl.handle.net/20.500.11794/24365

Gerbert, V., \& Durny, A. (2013). Analyse de l'activité collective en football. Une étude de cas avec les deux défenseurs centraux. Science \& Motricité, 79. doi.org/10.1051/sm/2012030.

Gréhaigne, J.-F. (2007). Configurations du jeu: débat d'idées \& apprentissage du football et des sports collectifs. Besançon : Presses de l'Université de Franche-Comté.

Gréhaigne, J.F. (Ed.). (2009). Autour du temps. Espaces, apprentissages, projets dans les sports collectifs. Besançon : Presses de l'Université de Franche-Comté.

Gréhaigne, J.-F., \& Godbout, P. (1995). Tactical knowledge in team sports from a constructivist and cognitivist perspective. Quest, 47, 490-505.

Gréhaigne, J.-F., \& Godbout, P. (1998). Observation, critical thinking and transformation: Three key elements for a constructivist perspective of the learning process in team sport. In R. S. Feingold, C. R. Rees, G. T. Barrette, L. Fiorentino, S. Virgilio, \& E. Kowalski (Eds.), Education for life (pp. 109-118). Garden City (NY): Adelphi University.

Gréhaigne, J.-F., \& Nadeau, L. (2015). L'enseignement et l'apprentissage de la tactique en sports collectifs : des précurseurs oubliés aux perspectives actuelles. eJRIEPS, 35, 106-140.

Gréhaigne, J.-F., Caty, D., \& Godbout, P. (2010). Modelling ball circulation in invasion team sports: a way to promote learning games through understanding. Physical Education and Sport Pedagogy, 15, (3), 257-270. Doi.10.1080/17408980903273139.

Gréhaigne, J.-F., Godbout, P., \& Zerai, Z. (2014). Analogies, configurations du jeu et intelligence tactique. In J.-F. Gréhaigne (Ed.) L'intelligence tactique. Des perceptions aux décisions tactiques en sports collectifs (pp. 301-314). Besançon : Presses de l'Université de Franche-Comté.

Gréhaigne, J.-F., Wallian, N., \& Brière-Guenoun, F. (2015). Débat d'idées, langage et interactions discursives et apprentissage des sports collectifs. eJRIEPS, Hors série 1, $1-23$.

Gréhaigne, J.F., Godbout, P., \& Mahut, N. (1999). L'enseignement des jeux par la compréhension : une revue de questions. STAPS, 48, 81-93.

Hopper, T., Butler, J., \& Storey, B. (2009). TGfU... Simply Good Pedagogy: Understanding a Complex Challenge. Ottawa: PHE Canada. 


\section{eJRIEPS spécial 3 janvier 2020}

Kermarrec, G. (2004). Stratégies d'apprentissage et autorégulation. Revue de question dans le domaine des habiletés sportives. Movement \& Sport Sciences, 53(3). doi:10.3917/sm.053.0009

Langevin, L. (1990). Le questionnement comme stratégie d'enseignement et d'apprentissage. Pédagogie collégiale, 4 (1), 12-13.

L'Écuyer, R. (1987). L'analyse de contenu : notion et étapes. In Deslauriers, J.-P. (dir.), Les méthodes de la recherche qualitative (pp. 49-65). Sillery, Québec : Les Presses de I'Université du Québec.

Maulini, O. (2005). Questionner pour enseigner et pour apprendre. Le rapport au savoir dans la classe. Issy-les-Moulineaux : ESF éditeur. http://archiveouverte.unige.ch/unige:34465

Nadeau, L., Georget, S., Fortier, K., \& Godbout, P. (2014). Savoirs collectifs, intelligence tactique et hockey sur glace. In J.-F. Gréhaigne (Ed.) L'intelligence tactique : Des perceptions à la logique ou non des décisions tactiques dans les sports collectifs. Besançon : Presses universitaires de Franche Comté.

Nadeau, L., Martel, D., Poulin-Beaulieu, I., \& Couture-Légaré, J. (2017, November). Players perceptions of effective/ineffective tactical situations in competitive ice hockey: A case study. Présentation orale au AIESEP International conference, Guadeloupe.

Platonov, V. N. (1988). L'entraînement sportif : théorie et méthodologie. Paris : éditions revue EPS.

Trilles, F. (1994). Les techniques et méthodes de l'entraînement sportif. Collection «Sports ». Paris : Éditions du CNFPT.

Werner, P., Thorpe, R., \& Bunker, D. (1996). Teaching games for understanding: Evolution of a model. Journal of Physical Education, Recreation and Dance, 67(1), 28-33.

Zerai, Z., Rezig, M. \& Zhigbi, M. (2008). Débats d'idées et apprentissage chez les filles en sport collectif. eJRIEPS, 13, 78-93. 